\title{
Note on generating all subsets of a finite set with disjoint unions
}

\author{
David Ellis \\ e-mail: dce27@cam.ac.uk
}

Submitted: Dec 2, 2008; Accepted: May 12, 2009; Published: May 20, 2009

Mathematics Subject Classification: 05D05

\begin{abstract}
We call a family $\mathcal{G} \subset \mathbb{P}[n]$ a $k$-generator of $\mathbb{P}[n]$ if every $x \subset[n]$ can be expressed as a union of at most $k$ disjoint sets in $\mathcal{G}$. Frein, Lévêque and Sebő [1] conjectured that for any $n \geq k$, such a family must be at least as large as the $k$-generator obtained by taking a partition of $[n]$ into classes of sizes as equal as possible, and taking the union of the power-sets of the classes. We generalize a theorem of Alon and Frankl [2] in order to show that for fixed $k$, any $k$-generator of $\mathbb{P}[n]$ must have size at least $k 2^{n / k}(1-o(1))$, thereby verifying the conjecture asymptotically for multiples of $k$.
\end{abstract}

\section{Introduction}

We call a family $\mathcal{G} \subset \mathbb{P}[n]$ a $k$-generator of $\mathbb{P}[n]$ if every $x \subset[n]$ can be expressed as a union of at most $k$ disjoint sets in $\mathcal{G}$. Frein, Lévêque and Sebő [1] conjectured that for any $n \geq k$, such a family must be at least as large as the $k$-generator

$$
\mathcal{F}_{n, k}:=\bigcup_{i=1}^{k} \mathbb{P} V_{i} \backslash\{\emptyset\}
$$

where $\left(V_{i}\right)$ is a partition of $[n]$ into $k$ classes of sizes as equal as possible. For $k=2$, removing the disjointness condition yields the stronger conjecture of Erdös - namely, if $\mathcal{G} \subset \mathbb{P}[n]$ is a family such that any subset of $[n]$ is a union (not necessarily disjoint) of at most two sets in $\mathcal{G}$, then $\mathcal{G}$ is at least as large as

$$
\mathcal{F}_{n, 2}=\mathbb{P} V_{1} \cup \mathbb{P} V_{2} \backslash\{\emptyset\}
$$

where $\left(V_{1}, V_{2}\right)$ is a partition of $[n]$ into two classes of sizes $\lfloor n / 2\rfloor$ and $\lceil n / 2\rceil$. We refer the reader to for example Füredi and Katona [5] for some results around the Erdös conjecture. In fact, Frein, Lévêque and Sebő [1] made the analagous conjecture for all $k$. (We call a 
family $\mathcal{G} \subset \mathbb{P}[n]$ a $k$-base of $\mathbb{P}[n]$ if every $x \subset[n]$ can be expressed as a union of at most $k$ sets in $\mathcal{G}$; they conjectured that for any $k \leq n$, any $k$-base of $\mathbb{P}[n]$ is at least as large as $\mathcal{F}_{n, k}$.)

In this paper, we show that for $k$ fixed, a $k$-generator must have size at least $k 2^{n / k}(1-$ $o(1))$; when $n$ is a multiple of $k$, this is asymptotic to $f(n, k)=\left|\mathcal{F}_{n, k}\right|=k\left(2^{n / k}-1\right)$. Our main tool is a generalization of a theorem of Alon and Frankl, proved via an Erdős-Stone type result.

As observed in [1], for a $k$-generator $\mathcal{G}$, we have the following trivial bound on $|\mathcal{G}|=m$. The number of ways of choosing at most $k$ sets in $\mathcal{G}$ must be at least the number of subsets of $[n]$, i.e.:

$$
\sum_{i=0}^{k}\left(\begin{array}{c}
m \\
i
\end{array}\right) \geq 2^{n}
$$

For fixed $k$, the number of subsets of $[n]$ of size at most $k-1$ is $\sum_{i=0}^{k-1}\left(\begin{array}{c}m \\ i\end{array}\right)=\Theta(1 / m)\left(\begin{array}{c}m \\ k\end{array}\right)$, so

$$
\sum_{i=0}^{k}\left(\begin{array}{c}
m \\
i
\end{array}\right)=(1+\Theta(1 / m))\left(\begin{array}{c}
m \\
k
\end{array}\right)=(1+\Theta(1 / m)) m^{k} / k !
$$

Hence,

$$
m \geq(k !)^{1 / k} 2^{n / k}(1-o(1))
$$

Notice that this ignores disjointness, and is therefore also a lower bound on the size of a $k$-base; it also ignores the fact that some unions may occur several times. We will improve the constant from $(k !)^{1 / k} \approx k / e$ to $k$ by taking into account disjointness. Namely, we will show that for any fixed $k \in \mathbb{N}$ and $\delta>0$, if $m \geq 2^{(1 /(k+1)+\delta) n}$, then any family $\mathcal{G} \subset \mathbb{P}[n]$ of size $m$ contains at most

$$
\left(\frac{k !}{k^{k}}+o(1)\right)\left(\begin{array}{c}
m \\
k
\end{array}\right)
$$

unordered $k$-tuples $\left\{A_{1}, \ldots, A_{k}\right\}$ of pairwise disjoint sets, where the $o(1)=o_{k, \delta}(1)$ term tends to 0 as $m \rightarrow \infty$ for fixed $k, \delta$. In other words, if we consider the 'Kneser graph' on $\mathbb{P}[n]$, with edge set consisting of the disjoint pairs of subsets, the density of $K_{k}$ 's in any sufficiently large $\mathcal{G} \subset \mathbb{P}[n]$ is at most $k ! / k^{k}+o(1)$. The proof uses an Erdös-Stone type result (Theorem 1) together with a result of Alon and Frankl (Lemma 4, which is Lemma 4.3 in $[2])$.

The $k=2$ case of this was proved by Alon and Frankl (Theorem 1.3 of [2]): for any fixed $\delta>0$, if $m \geq 2^{(1 / 3+\delta) n}$, then any family $\mathcal{G} \subset \mathbb{P}[n]$ of size $m$ contains at most

$$
\left(\frac{1}{2}+o(1)\right)\left(\begin{array}{c}
m \\
2
\end{array}\right)
$$

disjoint pairs, where the $o(1)$ term tends to 0 as $m \rightarrow \infty$ for fixed $\delta$. In other words, the edge-density in any sufficiently large subset of the Kneser graph is at most $\frac{1}{2}+o(1)$.

Our result will follow quickly from this. From the trivial bound above, any $k$-generator $\mathcal{G} \subset \mathbb{P}[n]$ has size $m \geq 2^{n / k}$, so putting $\delta=1 / k(k+1)$, we will see that the number of 
unordered $k$-tuples of pairwise disjoint sets in $\mathcal{G}$ is at most

$$
\left(\frac{k !}{k^{k}}+o(1)\right)\left(\begin{array}{l}
m \\
k
\end{array}\right)
$$

So

$$
2^{n} \leq\left(\frac{k !}{k^{k}}+o(1)+\Theta(1 / m)\right)\left(\begin{array}{c}
m \\
k
\end{array}\right)=\left(\frac{m}{k}\right)^{k}(1+o(1))
$$

and therefore

$$
m \geq k 2^{n / k}(1-o(1))
$$

where the $o(1)$ term tends to 0 as $n \rightarrow \infty$ for fixed $k \in \mathbb{N}$.

For $k=2$, this improves the estimate $m \geq \sqrt{2} 2^{n / 2}-1$ in [1] (Theorem 5.3) by a factor of $\sqrt{2}$. For $n$ even, it is asymptotically tight, but for $n$ odd, the conjectured smallest 2-generator (2) has size $(3 / \sqrt{2}) 2^{n / 2}-1$, so our constant is 'out' by a factor of $3 /(2 \sqrt{2})=1.061$ (to 3 d.p.)

For general $k$ and $n=q k+r$, the conjectured smallest $k$-generator (1) has size

$$
(k-r) 2^{q}+r 2^{q+1}-k=(k+r) 2^{-r / k} 2^{n / k}-k
$$

so our constant is out by a factor of $(1+r / k) 2^{-r / k} \leq 2^{1-1 / \ln 2} / \ln 2=1.061$ (to 3 d.p.).

It seems that different arguments will be required to improve the constant for $k \nmid n$, or to prove the exact result. Further, it seems likely that proving the same bounds for $k$ bases (i.e. without the assumption of disjoint unions) would be much harder, and require different techniques altogether.

\section{A preliminary Erdős-Stone type result}

We will need the following generalization of the Erdős-Stone theorem:

Theorem 1 Given $r \leq s \in \mathbb{N}$ and $\epsilon>0$, if $n$ is sufficiently large depending on $r, s$ and $\epsilon$, then any graph $G$ on $n$ vertices with at least

$$
\left(\frac{s(s-1)(s-2) \ldots(s-r+1)}{s^{r}}+\epsilon\right)\left(\begin{array}{l}
n \\
r
\end{array}\right)
$$

$K_{r}$ 's contains a copy of $K_{s+1}(t)$, where $t \geq C_{r, s, \epsilon} \log n$ for some constant $C_{r, s, \epsilon}$ depending on $r, s, \epsilon$.

Note that the density $\eta=\eta_{r, s}:=\frac{s(s-1)(s-2) \ldots(s-r+1)}{s^{r}}$ above is the density of $K_{r}$ 's in the $s$-partite Turán graph with classes of size $T, K_{s}(T)$, when $T$ is large.

Proof:

Let $G$ be a graph with $K_{r}$ density at least $\eta+\epsilon$; let $N$ be the number of $l$-subsets $U \subset V(G)$ 
such that $G[U]$ has $K_{r}$-density at least $\eta+\epsilon / 2$. Then, double counting the number of times an $l$-subset contains a $K_{r}$,

$$
N\left(\begin{array}{l}
l \\
r
\end{array}\right)+\left(\left(\begin{array}{l}
n \\
r
\end{array}\right)-N\right)(\eta+\epsilon / 2)\left(\begin{array}{l}
l \\
r
\end{array}\right) \geq(\eta+\epsilon)\left(\begin{array}{l}
n \\
r
\end{array}\right)\left(\begin{array}{l}
n-r \\
l-r
\end{array}\right)
$$

so rearranging,

$$
N \geq \frac{\epsilon / 2}{1-\eta-\epsilon / 2}\left(\begin{array}{l}
n \\
l
\end{array}\right) \geq \frac{\epsilon}{2}\left(\begin{array}{l}
n \\
l
\end{array}\right)
$$

Hence, there are at least $\frac{\epsilon}{2}\left(\begin{array}{l}n \\ l\end{array}\right) l$-sets $U$ such that $G[U]$ has $K_{r}$-density at least $\eta+\epsilon / 2$. But Erdös proved that the number of $K_{r}$ 's in a $K_{s+1}$-free graph on $l$ vertices is maximized by the $s$-partite Turán graph on $l$ vertices (Theorem 3 in [3]), so provided $l$ is chosen sufficiently large, each such $G[U]$ contains a $K_{s+1}$. Each $K_{s+1}$ in $G$ is contained in $\left(\begin{array}{c}n-s-1 \\ l-s-1\end{array}\right)$ $l$-sets, and therefore $G$ contains at least

$$
\frac{\epsilon}{2} \frac{\left(\begin{array}{c}
n \\
l
\end{array}\right)}{\left(\begin{array}{c}
n-s-1 \\
l-s-1
\end{array}\right)} \geq \frac{\epsilon}{2}(n / l)^{s+1}
$$

$K_{s+1}$ 's, i.e. a positive density of $K_{s+1}$ 's. Let $a=s+1, c=\frac{\epsilon}{2 l^{s+1}}$ and apply the following 'blow up' theorem of Nikiforov (a slight weakening of Theorem 1 in [4]):

Theorem 2 Let $a \geq 2, c^{a} \log n \geq 1$. Then any graph on $n$ vertices with at least $c n^{a} K_{a}$ 's contains a $K_{a}(t)$ with $t=\left\lfloor c^{a} \log n\right\rfloor$.

We see that provided $n$ is sufficiently large depending on $r, s$ and $\epsilon, G$ must contain a $K_{s+1}(t)$ for $t=\left\lfloor c^{s+1} \log n\right\rfloor=\left\lfloor\left(\frac{\epsilon}{2 l^{s+1}}\right)^{s+1} \log n\right\rfloor \geq C_{r, s, \epsilon} \log n$, proving Theorem 1.

\section{Density of $K_{k}$ 's in large subsets of the Kneser graph}

We are now ready for our main result, a generalization of Theorem 1.3 in [2]:

Theorem 3 For any fixed $k \in \mathbb{N}$ and $\delta>0$, if $m \geq 2^{\left(\frac{1}{k+1}+\delta\right) n}$, then any family $\mathcal{G} \subset \mathbb{P}[n]$ of size $|\mathcal{G}|=m$ contains at most

$$
\left(\frac{k !}{k^{k}}+o(1)\right)\left(\begin{array}{l}
m \\
k
\end{array}\right)
$$

unordered $k$-tuples $\left\{A_{1}, \ldots, A_{k}\right\}$ of pairwise disjoint sets, where the o(1) term tends to 0 as $m \rightarrow \infty$ for fixed $k, \delta$.

Proof:

By increasing $\delta$ if necessary, we may assume $m=2^{\left(\frac{1}{k+1}+\delta\right) n}$. Consider the subgraph $G$ of the 'Kneser graph' on $\mathbb{P}[n]$ induced on the set $\mathcal{G}$, i.e. the graph $G$ with vertex set $\mathcal{G}$ and edge set $\{x y: x \cap y=\emptyset\}$. Let $\epsilon>0$; we will show that if $n$ is sufficiently large depending 
on $k, \delta$ and $\epsilon$, the density of $K_{k}$ 's in $G$ is less than $\frac{k !}{k^{k}}+\epsilon$. Suppose the density of $K_{k}$ 's in $G$ is at least $\frac{k !}{k^{k}}+\epsilon$; we will obtain a contradiction for $n$ sufficiently large. Let $l=m^{f}$ (we will choose $f<\frac{\delta}{2(1+(k+1) \delta)}$ maximal such that $m^{f}$ is an integer). By the argument above, there are at least $\frac{\epsilon}{2}\left(\begin{array}{c}m \\ l\end{array}\right) l$-sets $U$ such that $G[U]$ has $K_{k^{-}}$-density at least $\frac{k !}{k^{k}}+\frac{\epsilon}{2}$. Provided $m$ is sufficiently large depending on $k, \delta$ and $\epsilon$, by Theorem 1 , each such $G[U]$ contains a copy of $K:=K_{k+1}(t)$ where $t \geq C_{k, k, \epsilon / 2} \log l=f C_{k, \epsilon}^{\prime} \log m=C_{k, \delta, \epsilon}^{\prime \prime} \log m$. Any copy of $K$ is contained in $\left(\begin{array}{c}m-(k+1) t \\ l-(k+1) t\end{array}\right) l$-sets, so $G$ must contain at least $\frac{\epsilon}{2} \frac{\left(\begin{array}{c}m \\ l\end{array}\right)}{\left(\begin{array}{c}m-(k+1) t \\ l-(k+1) t\end{array}\right)} \geq \frac{\epsilon}{2}(m / l)^{(k+1) t}$ copies of $K$.

But we also have the following lemma of Alon and Frankl (Lemma 4.3 in [2]), whose proof we include for completeness:

Lemma $4 G$ contains at most $(k+1) 2^{n(1-\delta t)}\left(\begin{array}{c}m \\ t\end{array}\right)^{k+1} \frac{1}{(k+1) !}$ copies of $K_{k+1}(t)$.

Proof:

The probability that a $t$-subset $\left\{A_{1}, \ldots, A_{t}\right\}$ chosen uniformly at random from $\mathcal{G}$ has union of size at most $\frac{n}{k+1}$ is at most

$$
\sum_{S \subset[n]:|S| \leq n /(k+1)}\left(\begin{array}{c}
2^{|S|} \\
t
\end{array}\right) /\left(\begin{array}{c}
m \\
t
\end{array}\right) \leq 2^{n}\left(2^{n /(k+1)} / m\right)^{t}=2^{n(1-\delta t)}
$$

Choose at random $k+1$ such $t$-sets; the probability that at least one has union of size at most $n /(k+1)$ is at most

$$
(k+1) 2^{n(1-\delta) t}
$$

But this condition holds if our $k+1 t$-sets are the vertex classes of a $K_{k+1}(t)$ in $G$. Hence, the number of copies of $K_{k+1}(t)$ in $G$ is at most

$$
(k+1) 2^{n(1-\delta t)}\left(\begin{array}{c}
m \\
t
\end{array}\right)^{k+1} \frac{1}{(k+1) !}
$$

as required.

If $m$ is sufficiently large depending on $k, \delta$ and $\epsilon$, we may certainly choose $t \geq\lceil 4 / \delta\rceil$, and comparing our two bounds gives

$$
\frac{\epsilon}{2}(m / l)^{(k+1) t} \leq(k+1) 2^{n(1-\delta t)}\left(\begin{array}{c}
m \\
t
\end{array}\right)^{k+1} \frac{1}{(k+1) !} \leq \frac{1}{2} 2^{n(1-\delta t)} m^{(k+1) t}
$$

Substituting in $l=m^{f}$, we get

$$
\epsilon \leq 2^{n(1-\delta t)} m^{f(k+1) t}
$$

Substituting in $m=2^{\left(\frac{1}{k+1}+\delta\right) n}$, we get

$$
\epsilon \leq 2^{n(1-t(\delta-f(1+(k+1) \delta)))} \leq 2^{-n}
$$


since we chose $f<\frac{\delta}{2(1+(k+1) \delta)}$ and $t \geq 4 / \delta$. This is a contradiction if $n$ is sufficiently large, proving Theorem 3.

As explained above, our result on $k$-generators quickly follows:

Theorem 5 For fixed $k \in \mathbb{N}$, any $k$-generator $\mathcal{G}$ of $\mathbb{P}[n]$ must contain at least $k 2^{n / k}(1-$ $o(1))$ sets.

Proof:

Let $\mathcal{G}$ be a $k$-generator of $\mathbb{P}[n]$, with $|\mathcal{G}|=m$. As observed in the introduction, the trivial bound gives $m \geq 2^{n / k}$, so applying Theorem 3 with $\delta=1 / k(k+1)$, we see that the number of ways of choosing $k$ pairwise disjoint sets in $\mathcal{G}$ is at most

$$
\left(\frac{k !}{k^{k}}+o(1)\right)\left(\begin{array}{c}
m \\
k
\end{array}\right)
$$

The number of ways of choosing less than $k$ pairwise disjoint sets is, very crudely, at most $\sum_{i=0}^{k-1}\left(\begin{array}{c}m \\ i\end{array}\right)=\Theta(1 / m)\left(\begin{array}{c}m \\ k\end{array}\right)$; since every subset of $[n]$ is a disjoint union of at most $k$ sets in $\mathcal{G}$, we obtain

$$
2^{n} \leq\left(\frac{k !}{k^{k}}+o(1)+\Theta(1 / m)\right)\left(\begin{array}{c}
m \\
k
\end{array}\right)=\left(\frac{m}{k}\right)^{k}(1+o(1))
$$

(where the o(1) term tends to 0 as $m \rightarrow \infty$ ), and therefore

$$
m \geq k 2^{n / k}(1-o(1))
$$

(where the $o(1)$ term tends to 0 as $n \rightarrow \infty$ ).

Note: The author wishes to thank Peter Keevash for bringing to his attention the result of Erdős in [3], after reading a previous draft of this paper in which a weaker, asymptotic version of Erdös' result was proved.

\section{References}

[1] Frein, Y., Lévêque, B., Sebő, A., Generating All Sets With Bounded Unions, Combinatorics, Probability and Computing 17 (2008) pp. 641-660

[2] Alon, N., Frankl, P., The Maximum Number of Disjoint Pairs in a Family of Subsets, Graphs and Combinatorics 1 (1985), pp. 13-21

[3] Erdös, P., On the number of complete subgraphs contained in certain graphs, Publ. Math. Inst. Hung. Acad. Sci., Ser. A 7 (1962), pp. 459-464

[4] Nikiforov, V., Graphs with many $r$-cliques have large complete $r$-partite subgraphs, Bulletin of the London Mathematical Society Volume 40, Issue 1 (2008) pp. 23-25

[5] Füredi, Z., Katona, G.O.H., 2-bases of quadruples, Combinatorics, Probability and Computing 15 (2006) pp. 131-141 